\title{
A NON-HAUSDORFF ASCOLI THEOREM FOR $k_{3}$-SPACES
}

\author{
GEOFFREY FOX AND PEDRO MORALES
}

\begin{abstract}
The paper establishes an Ascoli theorem in the space of continuous functions on a $k_{3}$-space to a regular space. The theorem, in terms of even continuity and the compact open topology $\tau_{c}$, properly contains the Ascoli theorems of Myers, Gale, Kelley-Morse, Bagley-Yang, and the $k_{3}$-space theorem of Noble.
\end{abstract}

1. Introduction. To date, there are, apparently, five important $k$-space Ascoli theorems. There is the more general theorem 4.2(i) of Noble [8, p. 403], containing his $k_{3}$-space Corollary 4.4. All of these theorems suppose the range space to be regular and Hausdorff, and the Hausdorff condition is essential in the direction of necessity. Our theorem assumes only regularity on the range space. As might be expected, the generalization of context requires, in the direction of necessity, a strict weakening of one of the usual Ascoli conditions. However, these weakened conditions turn out to be sufficient.

The basic instruments of the paper are an even continuity lemma for partial functions, and a separating equivalence relation for regular spaces. The terminology and facts used without specific reference are of Kelley [6].

2. $k_{3}$-spaces. Let $X=(X, \tau)$ be a topological space. The $k$-extension of $\tau$ is the family $k(\tau)$ of all subsets $U$ of $X$ such that $U \cap K$ is open in $K$ for every compact subset $K$ of $X$. It is clear that $k(\tau)$ is a topology on $X$ which is larger than $\tau$. A topological space $(X, \tau)$ is called a $k$-space if $\tau=k(\tau)[3$, p. 79]. Locally compact spaces and spaces satisfying the first countability axiom are familiar examples of $k$-spaces.

Let $X, Y$ be topological spaces. A function $f: X \rightarrow Y$ is called $k$-continuous if its restriction to each compact subset of $X$ is continuous [2, p. 245]. Henceforth, the family of $k$-continuous functions on $X$ to $Y$ will be denoted $C_{k}(X, Y)$ and the subfamily of continuous functions on $X$ to $Y$ will be denoted $C(X, Y)$. It is known that a topological space $X$ is a $k$-space if and only if $C_{k}(X, Y)=C(X, Y)$, for every topological space $Y$. A topological space $X$ is called a $k_{3}$-space if $C_{k}(X, Y)=C(X, Y)$ for every regular space

Received by the editors June 22, 1972 and, in revised form, November 16, 1972.

AMS (MOS) subject classifications (1970). Primary 54C35; Secondary 54D50.

Key words and phrases. $k$-space, $k_{3}$-space, even continuity, compact open topology, $R$-saturation. 
$Y$ [9, p. 195]. Thus, a $k$-space is a $k_{3}$-space, but not conversely, because the product of uncountably many copies of the real line, which is not a $k$-space, is a $k_{3}$-space [9, Theorem 5.6(i)].

Let $X, Y$ and $Z$ be nonempty sets. The symbol $\omega$ will denote the evaluation map $(f, x) \rightarrow f(x)$ on $Y^{X} \times X$ to $Y$, or any restriction of this map. An element $f$ of $Z^{X \times Y}$ determines the function $f: x \rightarrow f(x, \cdot)$ on $X$ to $Z^{Y}$. It is clear that the exponential map $\mu: f \rightarrow \tilde{f}$ is a bijection of $Z^{X \times Y}$ onto $\left(Z^{Y}\right)^{X}$.

The following lemma is contained implicitly in the proof of Theorem 1.2 in [8, p. 394]:

2.1 Lemma. Let $f \in C(X \times Y, Z)$. If $X$ is compact and $Z$ is regular, then the family $\{f(x, \cdot): x \in X\}$ is evenly continuous.

Let $X, Y$ and $Z$ be topological spaces. The symbol $S_{k}(X \times Y, Z)$ denotes the family of all functions $f \in Z^{X \times Y}$ such that, for each $x \in X$ and each compact subset $K$ of $Y$, the restrictions $f|\{x\} \times Y, f| X \times K$ are continuous.

2.2 TheOREM. Let $X$ be a $k_{3}$-space and let $Y$ be a regular space. Then every compact subset $F$ of $\left(C(X, Y), \tau_{c}\right)$ is evenly continuous.

Proof. Since $Y$ is regular, $\mu^{-1}\left(C\left(F,\left(C(X, Y), \tau_{c}\right)\right)\right) \subseteq S_{k}(F \times X, Y)$ [8, Theorem 3.1]. Further, since $F$ is compact and $X$ is a $k_{3}$-space, $S_{k}(F \times X, Y)=C(F \times X, Y)[8$, Theorem 3.4]. Let $\omega: F \times X \rightarrow Y$. Since $\mu(\omega): F \rightarrow\left(C(X, Y), \tau_{c}\right)$ is the inclusion map, it is continuous. Then $\omega$ is continuous, and therefore, by $2.1, F$ is evenly continuous.

3. A separating equivalence relation. Let $X$ be a topological space which is Hausdorff or regular. Consider the following equivalence relation on $X$ (reducing to equality when $X$ is Hausdorff): $x R y$ means that every open set containing $x$ contains $y$. For a subset $E$ of $X, E^{*}$ will denote the smallest $R$-saturated subset of $X$ containing $E$ ( $R$-saturation of $E)$. We will apply the following facts:

(1) Every closed set is $R$-saturated.

(2) If $x \in E^{*}$ there exists $y \in E$ such that $x R y$. In fact, if $x R y$ implies $y \notin E$, then $X-\{y: x R y\}$ is an $R$-saturated set containing $E^{*}$ but not $x$.

(3) If $E \subseteq A, B \subseteq E^{*}$ and $A$ is compact, then $B$ is compact. In fact, an open covering of $B$ is an open covering of $A$. It contains a finite subfamily covering $A$, which also covers $B$.

(4) If a net in $X$ converges to points $x, y \in X$, then $x R y$.

(5) If $K$ is compact, then $K^{*}$ is closed. In fact, if $x \in\left(K^{*}\right)^{-}$there is a net $N$ in $K^{*}$ converging to $x$. Since $K^{*}$ is compact, there is a subnet $N_{1}$ of $N$ converging to a point $y \in K^{*}$. Then $x R y$, so $x \in K^{*}$. 


\section{Ascoli theorem.}

4.1 THEOREM. Let $F \subseteq\left(C(X, Y), \tau_{c}\right)$. If $Y$ is regular, the following conditions are sufficient for the compactness of $F$ :

(a) $F^{*}$ is closed in $C(X, Y)$,

(b) $(F(x))^{-}$is compact for all $x \in X$, and

(c) $F$ is evenly continuous.

If, further, $X$ is a $k_{3}$-space, the conditions (a), (b) and (c) are necessary for the compactness of $F$.

Proof. Sufficiency. Let $\bar{F}$ be the closure of $F$ in $\left(Y^{X}, \tau_{p}\right)$, where $\tau_{p}$ is the pointwise topology. Then $\tau_{p}$ is jointly continuous on $\bar{F}$; therefore $\bar{F} \subseteq C(X, Y)$ and $\tau_{p}=\tau_{c}$ on $\bar{F}$. Since $\bar{F} \subseteq \prod_{x \in X}(F(x))^{-}$, (b) implies that $\bar{F}$ is $\tau_{p}$-compact, therefore $\tau_{c}$-compact. Let $\hat{F}$ denote the closure of $F$ in $\left(Y^{X}, \tau_{c}\right)$, so that $F \subseteq \hat{F} \subseteq \bar{F}$. Since $\hat{F}$ is closed in $\left(\bar{F}, \tau_{c}\right)=\left(\bar{F}, \tau_{p}\right), \hat{F}$ is closed in $\left(Y^{X}, \tau_{p}\right)$, and therefore $\hat{F}=\bar{F}$. Thus $\hat{F}$ is the closure of $F$ in $\left(C(X, Y), \tau_{c}\right)$, so that (a) implies $F \subseteq \hat{F} \subseteq F^{*}$. It follows that $F$ is $\tau_{c}$-compact.

Necessity. Since $F$ is $\tau_{c}$-compact, $F^{*}$ is closed in $\left(C(X, Y), \tau_{c}\right)$. For $x \in X, F(x)$ is compact, therefore $(F(x))^{-}$is compact (because $Y$ is regular). By $2.2, F$ is evenly continuous.

REMARKS. (1) If $Y$ is regular, the condition (a) of (4.1) is weaker than the classical condition:

(a') $F$ is closed in $C(X, Y)$.

If $Y$ is Hausdorff, (a) coincides with $\left(\mathrm{a}^{\prime}\right)$.

(2) If $Y$ is regular and non-Hausdorff, (a) is strictly weaker than $\left(a^{\prime}\right)$. In fact, $\left(C(X, Y), \tau_{c}\right)$ is regular and non-Hausdorff, therefore not $T_{1}$. So there exists $f \in C(X, Y)$ such that $\{f\} \subset(\{f\})^{-} \subseteq\{f\}^{*}$. Then $F=\{f\}$ satisfies (a) but not $\left(a^{\prime}\right)$. Consequently, if we read $\left(a^{\prime}\right)$ for (a) in 4.1 , the theorem would be weakened in the sufficiency direction, and rendered false in the necessity direction.

(3) Theorem 4.1 strictly contains the Corollary 4.4 of Noble [8, p. 403], the Ascoli theorem of Myers (stated implicitly in his Theorem 4.1) [7, pp. 497-498], the Ascoli theorems of Bagley-Yang [1, pp. 704-705], and the Ascoli theorem of Kelley-Morse [6, p. 236]. Because of Theorems (3.8) and (3.8a) of Poppe [10, pp. 115-116], 4.1 strictly contains the Ascoli theorem of Gale [5, p. 304].

\section{REFERENCES}

1. R. W. Bagley and Y. S. Yang, On k-spaces and function spaces, Proc. Amer. Math. Soc. 17 (1966), 703-705. MR 33 \#693.

2. R. Brown, Function spaces and product topologies, Quart. J. Math. Oxford Ser. (2) 15 (1964), 238-250. MR 29 \#2779.

3. D. E. Cohen, Spaces with weak topology, Quart. J. Math. Oxford Ser. (2) 5 (1954), 77-80. MR 16, 62. 
4. R. H. Fox, On topologies for function spaces, Bull. Amer. Math. Soc. 51 (1945), 429-432. MR 6, 278.

5. D. Gale, Compact sets of functions and function rings, Proc. Amer. Math. Soc. 1 (1950), 303-308. MR 12, 119.

6. J. Kelley, General topology, Van Nostrand, Princeton, N.J., 1955. MR 16, 1136.

7. S. B. Myers, Equicontinuous sets of mappings, Ann. of Math. (2) 47 (1946), 496-502. MR 8, 165.

8. N. Noble, Ascoli theorems and the exponential map, Trans. Amer. Math. Soc. 143 (1969), 393-411. MR 40 \#1978.

9. - - The continuity of functions on Cartesian products, Trans. Amer. Math. Soc. 149 (1970), 187-198. MR 41 \#2636.

10. H. Poppe, Stetige Konvergenz und der Satz von Ascoli und Arzelà, Math. Nachr. 30 (1965), 87-122. MR 32 \#6400.

Département de Mathématiques, Université de Montréal, Montréal, Québec, CANADA 